\title{
Epicurus in the Enlightenment, Edited by Neven Leddy and Avi S. Lifschitz
}

\section{Maurizio Melai}

\section{(2) OpenEdition}

1 Journals

\section{Edizione digitale}

URL: http://journals.openedition.org/studifrancesi/6286

DOI: 10.4000/studifrancesi.6286

ISSN: 2421-5856

\section{Editore}

Rosenberg \& Sellier

\section{Edizione cartacea}

Data di pubblicazione: 1 novembre 2010

Paginazione: 551-552

ISSN: 0039-2944

\section{Notizia bibliografica digitale}

Maurizio Melai, «Epicurus in the Enlightenment, Edited by Neven Leddy and Avi S. Lifschitz», Studi

Francesi [Online], 162 (LIV | III) | 2010, online dal 30 novembre 2015, consultato il 09 janvier 2021. URL: http://journals.openedition.org/studifrancesi/6286 ; DOI: https://doi.org/10.4000/studifrancesi.6286

Questo documento è stato generato automaticamente il 9 janvier 2021.

\section{(c) (i) (9)}

Studi Francesi è distribuita con Licenza Creative Commons Attribuzione - Non commerciale - Non opere derivate 4.0 Internazionale. 


\title{
Epicurus in the Enlightenment, Edited by Neven Leddy and Avi S. Lifschitz
}

\author{
Maurizio Melai
}

\section{NOTIZIA}

Epicurus in the Enlightenment, Edited by Neven LEDDY and Avi S. LIFSCHITZ, Oxford, Voltaire Foundation, 2009 («SVEC» 2009:12), pp. 258.

1 Tramite un approccio interdisciplinare, che coinvolge questioni di etica, di religione, di politica e di scienze naturali, il presente volume esplora le modalità e le strategie, estremamente complesse, attraverso le quali l'Illuminismo europeo reinterpreta l'antica filosofia epicurea. La critica novecentesca si è troppo spesso dilettata in maniera semplicistica, concentrandosi sui temi del materialismo e dell'edonismo, a reperire $i$ tratti pre-illuministici dell'Epicureismo o i tratti neo-epicurei dell'Illuminismo. Il carattere eterogeneo dei contributi che compongono il presente studio riflette, scongiurando il rischio di approssimazione critica, la complessità del rapporto dialettico che il pensiero illuministico instaura con la filosofia di Epicuro. Tale complessità è dovuta in primo luogo al carattere composito della dottrina epicurea: gli intellettuali settecenteschi possono, di conseguenza, separarne i vari aspetti, riutilizzarne - spesso in maniera parziale e strumentale - soltanto alcuni, smembrare la coerenza complessiva del sistema filosofico antico per recuperarne quegli elementi che meglio si adattano alla loro argomentazione moderna. È così che, all'interno del pensiero illuministico, vari aspetti provenienti dalla tradizione epicurea si incrociano in modo sincretico con elementi relativi alla tradizione cristiana, allo spinozismo, o ancora allo stoicismo e all'agostinismo.

2 Il Settecento compie un'appropriazione selettiva di temi epicurei, attualizzandoli e mettendoli in continua interazione con le conquiste del pensiero moderno. Di tale appropriazione, più o meno debita e legittima, si occupano i vari articoli di Epicurus in the Enlightenment. In particolare, il contributo di Elodie ARGAUD (Bayle's defense of 
Epicurus: the use and abuse of Malebranche's "Méditations chrétiennes", pp. 13-30) mostra come Bayle, nell'articolo «Epicure» del suo Dictionnaire historique et critique, si serva della teoria anti-provvidenziale epicurea per dare un'interpretazione assai discutibile e tendenziosa del pensiero di Malebranche. L'articolo di Hans W. BLOM, The Epicurean motif in Dutch notions of sociability in the seventeenth century (pp. 31-51) si sofferma sul precetto epicureo del perseguimento dell'interesse personale e sull'importanza che esso ricopre nel dibattito filosofico olandese da Lipsius fino a Grotius, Spinoza e Mandeville. Il contributo di Thomas AHNERT, Epicureanism and transformation of natural law in the early German Enlightenment (pp. 53-68), si interessa della ricezione tedesca dell'Epicureismo e dell'influenza che esso esercita sulle teorie relative alla legge naturale elaborate, tra il 1670 ed il 1750, da intellettuali come Pufendorf, Thomasius e Schmauss. Charles T. WOLFE, in A happiness fit for organic bodies: La Mettrie's medical Epicureanism (pp. 69-83), individua le connessioni che intercorrono tra materialismo ed etica edonistica nel pensiero di La Mettrie, mentre Natania MEEKER, in Sexing Epicurean materialism in Diderot (pp. 85-104), mette in evidenza il ruolo svolto dalla nozione di genere e dal paradigma della femminilità nel recupero diderotiano del materialismo epicureo. Nell'articolo Helvétius as an Epicurean political theorist (pp. 105-118), Pierre FORCE mostra come il riferimento alla tradizione epicurea sia la chiave per cogliere la coerenza del pensiero politico di Helvétius, mentre Andrew KAHN, in Epicureanism in the Russian Enlightenment: Dmitrii Anichkov and atomic theory (pp. 119-136), mette in evidenza i tratti epicurei che caratterizzano le teorie atomistiche e vitalistiche dei naturalisti russi Mikhail Lomonosov e Dmitrii Anichov. Interessandosi alla presenza dell'Epicureismo nella filosofia inglese del Settecento, Matthew NIBLETT, autore dell'articolo Man, morals and matter: Epicurus and materialist thought in England from John Toland to Joseph Priestley (pp. 137-159), riflette sul percorso di attenuazione delle teorie materialiste che si riscontra nel passaggio dalla filosofia radicale di Toland a quella deista di Priestley. Il contributo di James A. HARRIS, The Epicurean in Hume (pp. 161-181), e quello di Neven LEDDY, Adam Smith's critique of Enlightenment Epicureanism (pp. 183-205), si focalizzano rispettivamente sul confronto dialettico che David Hume e Adam Smith instaurano, in diversi dei loro scritti, con la dottrina di Epicuro. Infine, Avi S. LIFSCHITZ, nell'articolo The Enlightenment revival of the Epicurean history of language and civilisation (pp. 207-226), mostra come la teoria epicurea sull'origine del linguaggio, nata come alternativa sia alla teoria platonica che a quella aristotelica, sia discussa e riutilizzata da filosofi moderni del calibro di Leibniz, Vico, Condillac e Mendelssohn. 